\title{
Histological and micro-computed tomographic observations after maxillary sinus augmentation with porous hydroxyapatite alloplasts: a clinical case series
}

\author{
Hidemi Nakata ${ }^{1,2}$, Shinji Kuroda ${ }^{2 *}$, Noriko Tachikawa², Emi Okada², Maho Akatsuka², Shohei Kasugai ${ }^{2}$ \\ and Hisatomo Kondo ${ }^{1,2}$
}

\begin{abstract}
Background: It is important to visualize the phenomenon which occurs in actual clinical cases to decide the timing of implant placement subsequently after sinus graft. Although several clinical cases of bone augmentation using xenograft have been evaluated, the number of those reports which have described bone remodeling by alloplasty are not sufficient.

Findings: In the present report, to investigate bone remodeling histologically after maxillary sinus augmentation with porous hydroxyapatite alloplast, bone cores from the sinus floor of three female nonsmoking patients (aged 64-73 years) were collected 6 months after sinus lift surgery, embedded in methyl methacrylate resin, and prepared by conventional methods. Bone architecture and graft residues were evaluated by micro-computed tomography of the same specimens. Hematoxylin-eosin and Villanueva-Goldner staining revealed mature osteoblasts and multinucleated osteoclasts on the grafted sinus floor and surface of residual hydroxyapatite particles. The particulate interspace was partially filled with osteoid and calcified tissue and showed active vascularization.
\end{abstract}

Conclusion: The results suggested that bone regeneration and angiogenesis within and between porous hydroxyapatite particles were sufficiently found after 6 month histologically in the grafted sinus floor.

Keywords: Sinus lift, Alloplast, Bone regeneration, Micro-computed tomography, Porous hydroxyapatite

\section{Background}

Porous hydroxyapatite is a major bone substitute (Yoshikawa et al. 2009; Yuasa et al. 2004). Its high pore rate (72-78 \%) and large interpore distance markedly reduce its mechanical strength (Moller et al. 2014; Liu 1996). However, greater porosity facilitates intraparticulate cell migration from adjacent tissues (Phipps et al. 2012; Fassina et al. 2010). Bone conductivity of hydroxyapatite also induces bone formation in the particles (Campana et al. 2014). Hydroxyapatite particles can be used to

\footnotetext{
*Correspondence: skuroda.mfc@tmd.ac.jp

${ }^{2}$ Department Oral Implantology and Regenerative Dental Medicine, Graduate School of Medical and Dental Sciences, Tokyo Medical and Dental University, 1-5-45, Bunkyo-ku, Yushima, Tokyo 113-8510, Japan Full list of author information is available at the end of the article
}

maintain the lifted sinus space and promote mineralization and maturation of new bone; however histological and radiographic findings of bone regeneration clinically induced by porous hydroxyapatite alloplast have not been frequently reported (Kolerman et al. 2012). Difficulty in the surgical procedure can be a major reason why only a few number of this kind of works have been reported, wherein just a single drilling in the jaw with a trephine bur is allowed to retrieve the bone-with- HA sample, simultaneously preparing the direction and cavity diameter for the implant at once. And it is often that mishandling with the drill bit would not be recovered, resulting in missing of the primary stabilization of implants. Furthermore, it might not always be easy to obtain patients' understanding of the unusual methodology of the

\section{Springer}


implant surgery and eligible to proceed with it for benefit to patients.

In this study, bone cores from the sinus floor of three patients were collected 6 months after maxillary sinus augmentation with porous hydroxyapatite alloplast to investigate bone formation histologically and evaluate bone architecture and graft residues by micro-computed tomography $(\mathrm{CT})$.

\section{Methods}

All experiments were approved (approval number 884) by and followed the guidelines of Tokyo Medical and Dental University.

\section{Patients}

Three female patients (aged 64-73 years) with severe alveolar bone resorption originating from periodontal disease or trauma were enrolled in this study. None smoked or had systemic pathologies affecting the immune system and contraindications for surgery and sinus grafting. Preoperative CT revealed clear maxillary sinuses and bone height from the alveolar ridge to the sinus floor of 1-2 mm (Fig. 1a). All the patients gave written consent for bone removal during dental implant surgery and its analysis.

The patients underwent sinus lift surgery under sedation and local anesthesia from the lateral approach according to the protocol described by Boyne and James (1980). After sinus membrane elevation, porous hydroxyapatite particles $(\phi 1-2 \mathrm{~mm}, 1 \mathrm{~g}$; NeoBone, Covalent Materials Corporation, Tokyo, Japan) were grafted onto the sinus floor from the lateral window (Fig. 1b).

\section{Collection of bone core and implant placement}

Six months after sinus lift surgery, a full-thickness flap was raised and the sites for dental implant placement were marked with a $\phi 2-\mathrm{mm}$ round bur to a depth of approximately $2 \mathrm{~mm}$ to remove the residual cortical bone in collected bone core. Bone cores were removed until approximately $6 \mathrm{~mm}$ vertically from the alveolar ridge by using a trephine bur (3-mm outer diameter), and dental implants (Astratech Implant System [4.0ST $\times 9 \mathrm{~mm}$, Dentsply Implants, Mannheim, Germany, Brånemark System MkIII TiU $[\phi 3.75 \times 10 \mathrm{~mm}]$ or NobelReplace Tapered $[\phi 4.3 \times 10 \mathrm{~mm}]$, Nobel Biocare, Göthenburg, Sweden) were placed into the sockets. The stabilization torque during placement was around $15-20 \mathrm{Ncm}$. All the implants were submerged with 0 -mm-high cover screws.

\section{Specimen preparation}

The bone cores were immediately fixed in $10 \%$ paraformaldehyde ( $\mathrm{pH} 7.3$ ), dehydrated in 70-99.5\% ethanol and acetone for 1-2 days at each stage, exposed to a 1:1 ratio of acetone and methyl methacrylate (MMA) monomer for 3 days, further treated with MMA monomer for 10 days, embedded in MMA resin, and allowed to harden for 12 days. They were then cut by using a precision sectioning saw (IsoMet-1000, Buehler, Lake Bluff, IL) and sliced into $6-\mu \mathrm{m}$-thick sections (nondecalcified) with a rotary microtome (Leica RM2255, Leica Biosystems, Wetzlar, Germany).

\section{Histological analysis}

Sections were deresinated with xylene for $2 \mathrm{~h}$ at $60{ }^{\circ} \mathrm{C}$. For Villanueva-Goldner staining, deresinated sections were stained with iron hematoxylin for $20 \mathrm{~min}$, treated with $1 \%$ hydrochloric acid-ethanol solution, stained with Ponceau fuchsin for $120 \mathrm{~min}$, treated with $1 \%$ acetic acid, and stained with phosphotungstic acid-phosphomolybdic acid solution. They were then stained with naphthol green solution for $30 \mathrm{~min}$ and washed with 70-99.5 \% ethanol. For hematoxylin-eosin staining, deresinated sections were stained with iron hematoxylin for $20 \mathrm{~min}$, treated with $1 \%$ hydrochloric acid-ethanol solution, stained with eosin solution, and washed with $99.5 \%$ ethanol. After treatment with xylene, all the stained sections were enclosed and observed with a light microscope (Olympus, Tokyo, Japan).

\section{Micro-CT analysis}

The bone cores were also scanned with a microfocus X-ray CT system (Scan Xmate-L090, Comscantecno Co., Ltd., Yokohama, Japan). The X-ray source was set at $80 \mathrm{kV}$ and $100 \mu \mathrm{A}$, and the samples were rotated $360^{\circ}$. Image resolution was fixed at a pixel size of $11.587 \mu \mathrm{m}$. The magnification was 8.630 , and slice thickness was $11.587 \mu \mathrm{m}$. Three-dimensional measurements and structural analyses were performed with custom software (TRI/3D-Bon, Ratoc System Engineering, Kanagawa, Japan). The images were binarized with a threshold range between 1 and 255 (gray values). To distinguish grafted and native bone from the background, a suitable threshold range is required for grafted bone: the minimum value was 31 and gradation range was 224 for native bone with hydroxyapatite particles, and the minimum value was 123 and gradation range was 132 for hydroxyapatite particles alone. The parameters included percentage of bone volume (BV), bone surface to bone volume (BS/ $\mathrm{BV})$, bone volume-to-tissue volume (BV/TV), trabecular thickness (Tb.Th), trabecular number (Tb.N), trabecular separation (Tb.Sp), trabecular spacing (Tb.Spac), and fractal dimension (D) were analyzed. BV/TV is the percentage of BV relative to TV within the bone core. Tb.Th represents the mean thickness of individual trabeculae, 


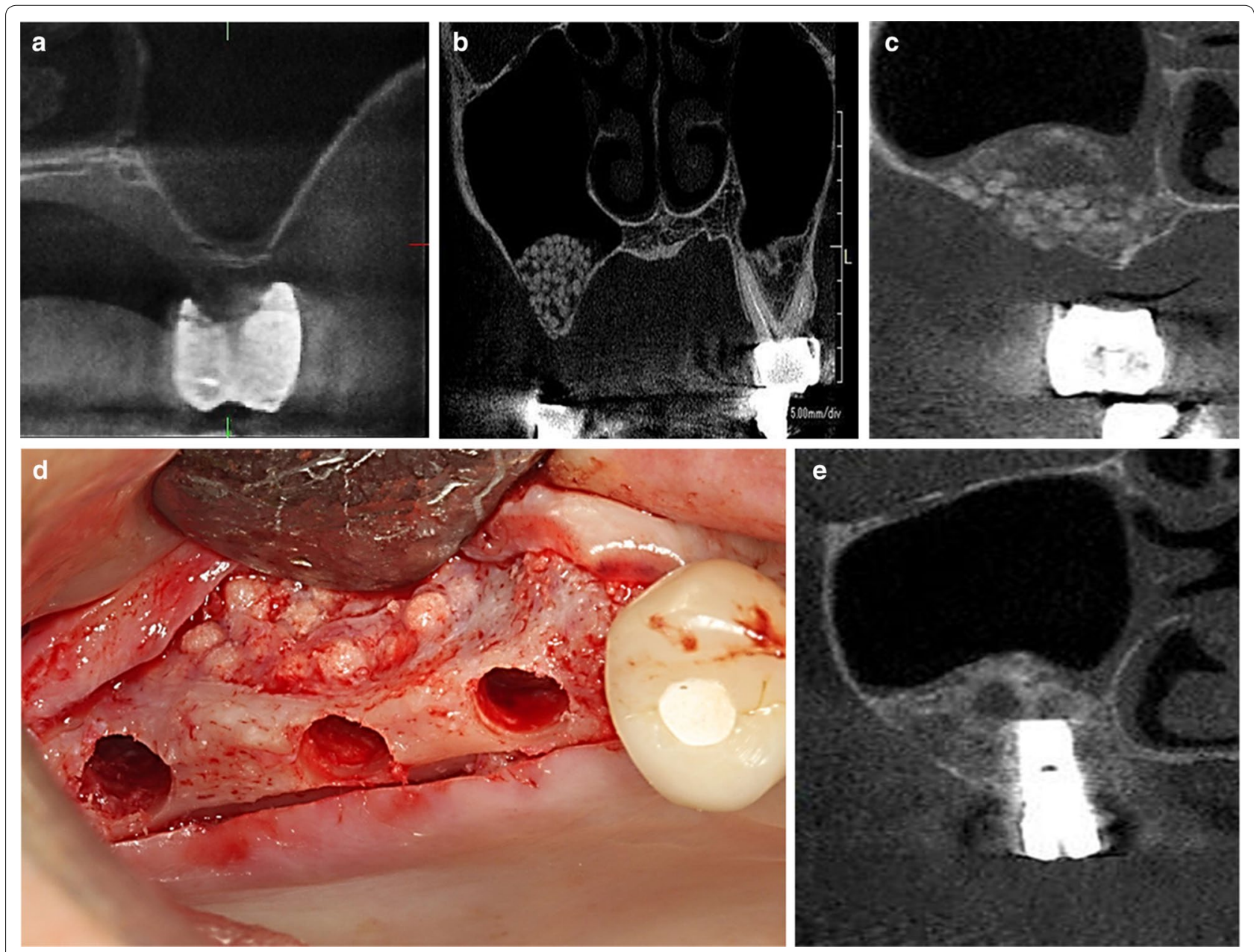

Fig. 1 CT scan before sinus graft. The original bone thickness from the alveolar ridge to the sinus floor was approximately 1-2 mm in average (a). CT image after maxillary sinus augmentation (b). A postoperative image for estimating the dental implant placement site (c). Photograph obtained after harvesting the bone cores and preparation for dental implant placement (d). Hydroxyapatite particles are visible in the lateral window. The operated sinus floors did not shown signs of inflammation. Scale $1 \mathrm{~mm}$

and Tb.Sp shows the space between trabeculae within a sample. Tb.Pf was developed by Hahn et al. (Browaeys et al. 2007): lower Tb.Pf signifies better connected trabecular lattices, while higher Tb.Pf indicates more disconnected trabecular structure.

\section{Results}

\section{Surgical outcomes}

The postoperative course was uneventful, and the amount of hard tissue was sufficient for dental implant placement evaluated by preoperative CT (Fig. 1c). The stability of the bone substitute was favorable (Fig. 1d). The implants showed around $20-25 \mathrm{Ncm}$ of torque for placement, and were stabilized enough to resist the movement which prevent osseointegration of the dental implants during healing periods (Fig. 1e).

\section{Histological findings}

Each bone core represents different histological view (Fig. 2a, 1-3). The lower side indicates the alveolar ridge in Fig. 2a 1-3. Numerous fibroblasts (FB) which supposed to differentiate into osteoblasts migrated into the surface of pores of hydroxyapatite particles, and newly formed bone (NB) was observed with mature multinucleated osteoclast $(\mathrm{OC})$ at the interspace of hydroxyapatite residues (HA) (Fig. 2b). Activated mature osteoblasts (OB) with secretory vesicles were located at the surface of osteoid (red) which engrails the newly formed bone (green) (Fig. 2c-1: low magnification, Fig. 2c-2: high magnification). Bone formation has been taken place along the interface of the hydroxyapatite pores (Fig. 2d). New blood vessels (V: vein, A: artery) were also found in the particles and surrounding areas (Fig. 2d, e). Osteoclasts 

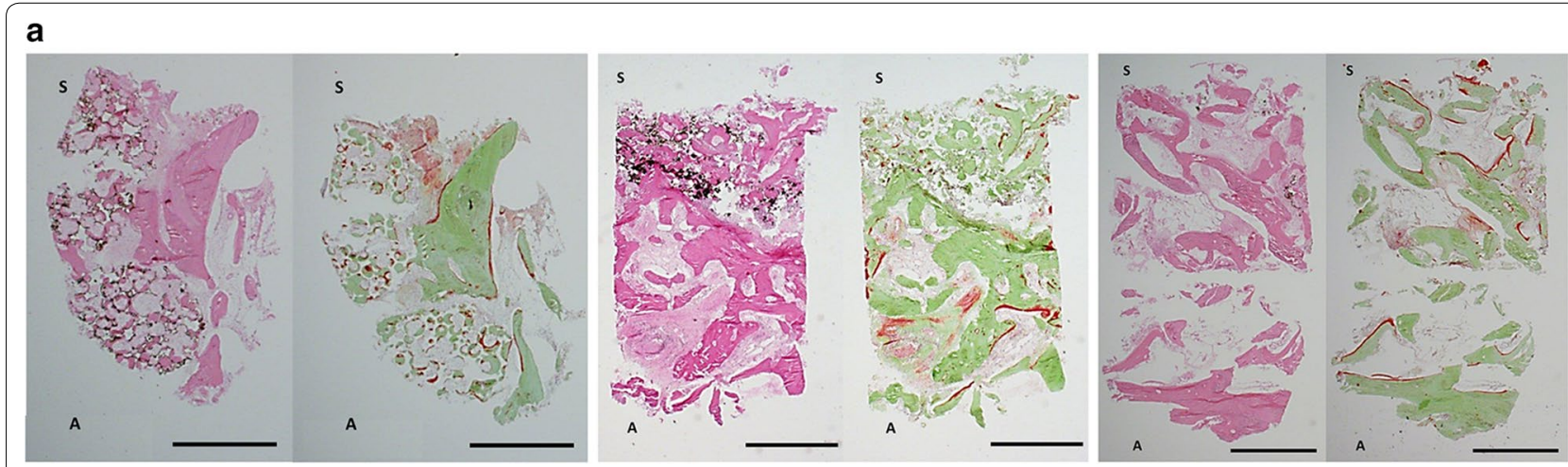

b

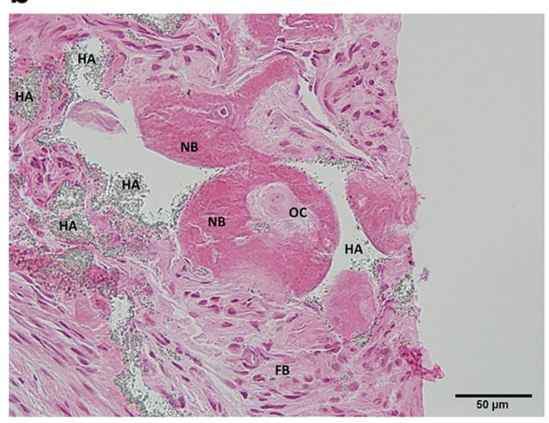

C
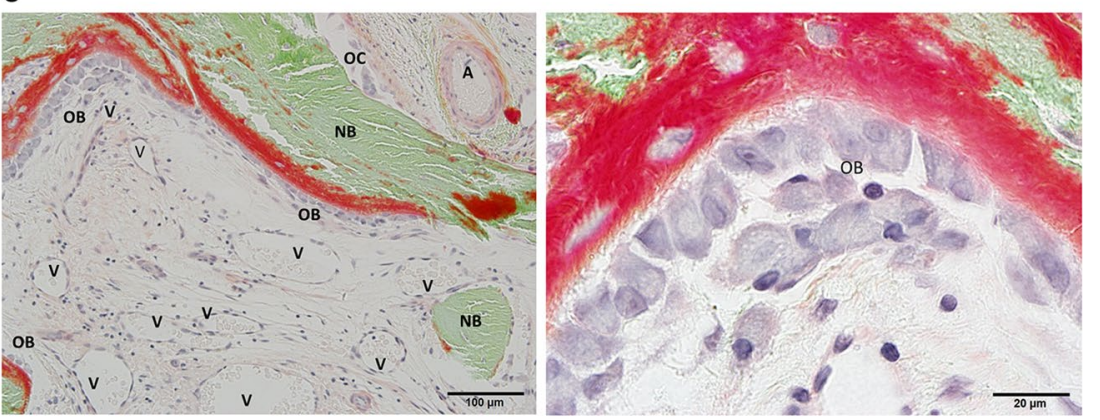

d

e
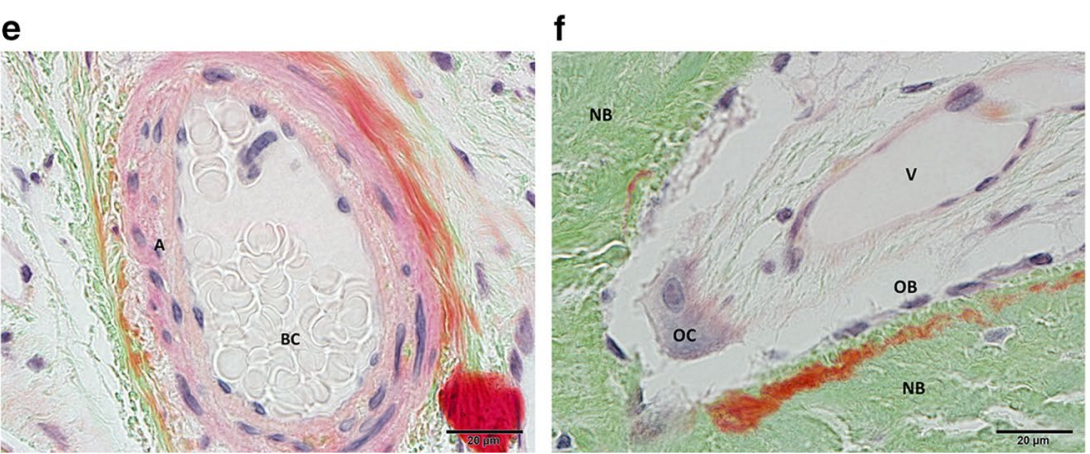

Fig. 2 Low-magnification (x 40) images of stained vertical sections of whole bone blocks. (a 1-3; S Snus floor. A Alveolar ridge). The dark brown coloration indicates residual hydroxyapatite in sections stained with hematoxylin-eosin (left). Bone and osteoid are indicated by green and red, respectively, in sections stained with Villanueva-Goldner trichrome (right). b has been shown high-magnification images of a-2. Residual hydroxyapatite $(\mathrm{HA})$, migration of numerous fibroblasts (FB), and bone formation (NB) have been shown ( $\times 200$; hematoxylin-eosin stain). An osteoclast (OC) is visible in bone formed in the pores of hydroxyapatite particles. Active vascularization ( $V$ vein, A Artery) and activated mature osteoblasts (OB) showing polygonal shape and numerous secretory vesicles on the osteoid surface (red) can be seen in c $(1: \times 100,2: \times 1000$; Villanueva-Goldner stain). High magnification of $\mathbf{a}-2$ indicated in $\mathbf{d}$ : Globular bone formation (NB) in the pores of hydroxyapatite particles has been shown ( $\times 200$; VillanuevaGoldner stain). Small arteries showing blood cells were observed (e: $\times 1000$; Villanueva-Goldner stain). A multinuclear large osteoclast was observed with osteoblasts and vascularization on the surface of new bone $(\mathbf{f}: \times 1000$; Villanueva-Goldner stain)

(OC) that found at the interface of new bone (NB; green) and hydroxyapatite (blank) may absorb the surface of particles (Fig. 2f).

\section{Micro-CT findings}

The architecture of the bone cores was different between each other and the volume of residual hydroxyapatite (purple) also varied among the patients (Fig. 3a-c, Additional files 1,2,3). The lower side indicates the alveolar ridge in Fig. 3a-c. One case shows abundant residues of hydroxyapatite from the alveolar ridge to the bottom of the bone core (Fig. 3a), whereas, hydroxyapatite residues were located at the distal region from the alveolar ridge in two cases (Fig. 3b, c). There can be seen residual bone in the right side of the bone core, and also observed outgrowth of the bone structure from the original bone into the hydroxyapatite particulate interspace (Fig. 3a). New bone and osteoid were appeared as a blanched area in all 
a

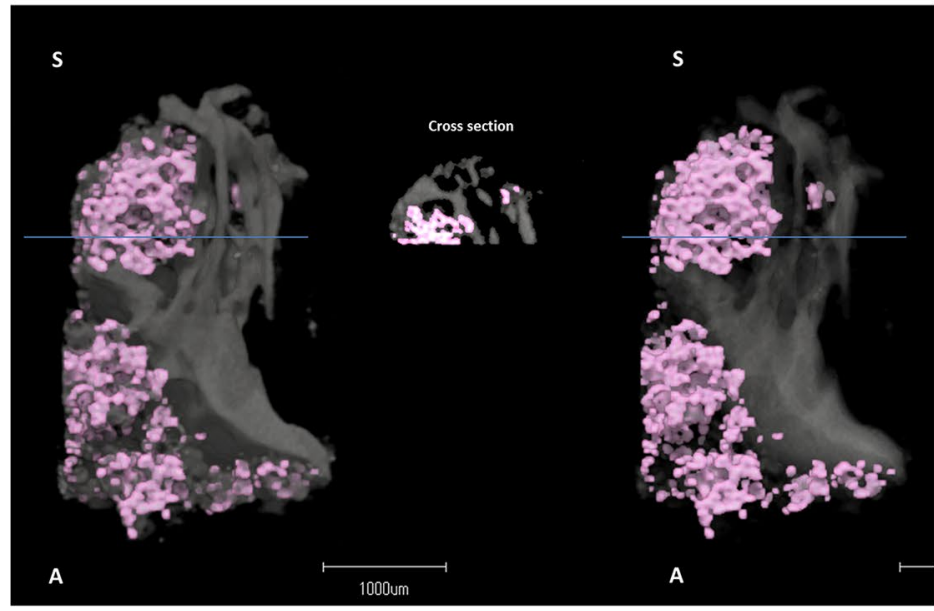

b

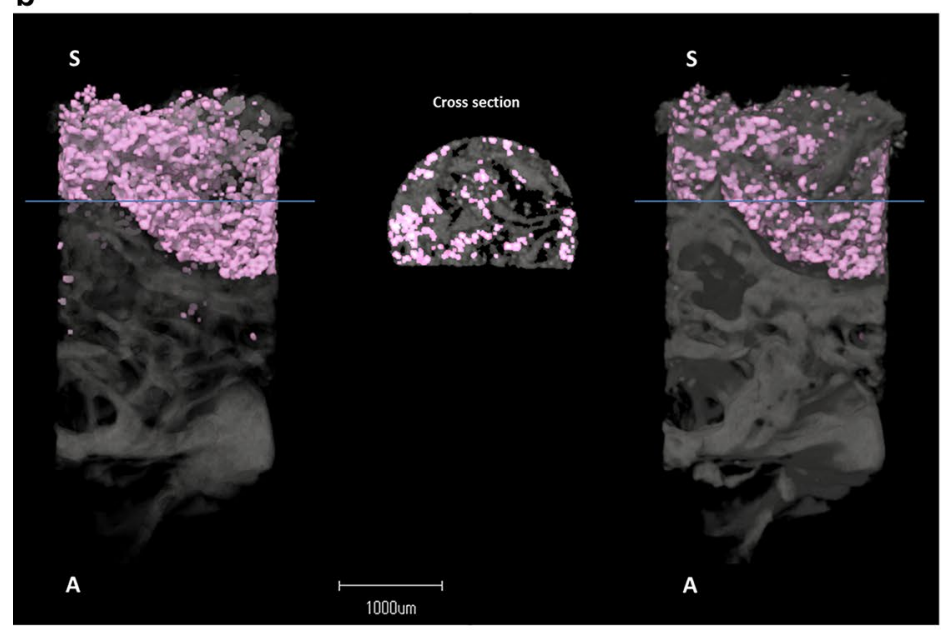

C

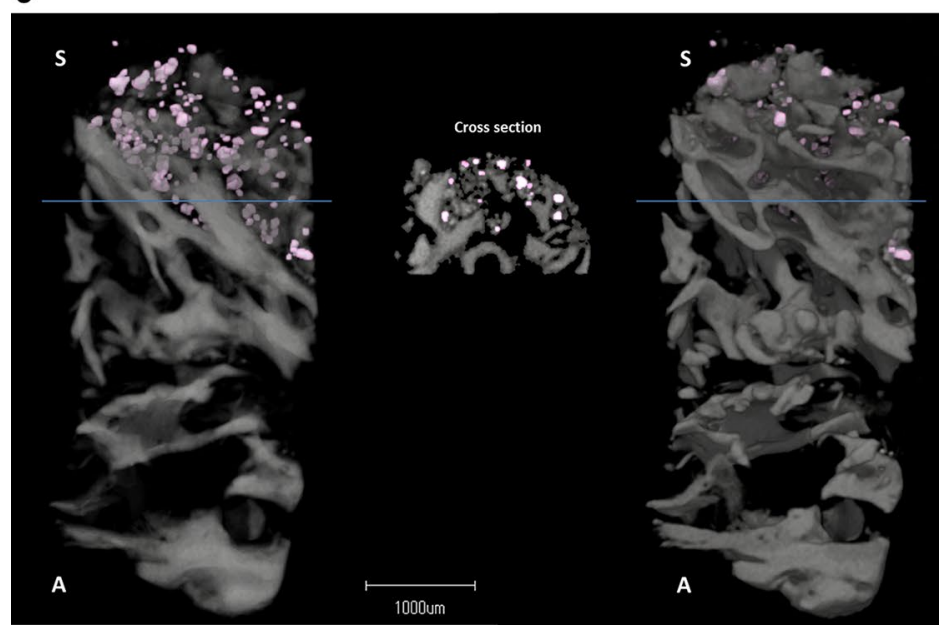

Fig. 3 Micro-CT scans of trabecular bone. Vertical 3-dimensional images and cross-sectional 2-dimentional images at the level of the blue line (a-c; S Snus floor. A Alveolar ridge) and movie of horizontal rotation (Additional files 1,2,3) of the bone core are shown. A fixed threshold range (123-132) was applied to segment the grafted bone from the background. New bone is observable in the grafted sites. Residual hydroxyapatite is indicated in purple; its volume was not equal in the samples 
the patients (Fig. 3a-c, cross sections; middle of the figures). The radiographic data are summarized in Table 1. The mean BV/TV was approximately $30 \%$, and mean hydroxyapatite residue was $1.2 \%$ (Table 1 ). The residual original bone area of Fig. 3a was avoided for the calculation and analysis in this study to evaluate only the grafted area.

\section{Discussion}

In the present study, bone formation and vascularization were observed 6 months after sinus augmentation with porous hydroxyapatite alloplast. These results confirmed the evidence that 6 month considered as a standard healing period subsequently to sinus grafting would be sufficient for preparation of implant placement (Tatum 1986). Kurabuda, et al. described differences of newly formed bone/residual material ratio between 3 kinds of graft materials; demineralized freeze-dried bone powder (DFBP), deproteinized bovine bone (DBBG), and porous hydroxyapatite (PHA) at 6 month after sinus graft, and their results showed that the lowest rate of newly formed bone was observed in patients who received PHA (Karabuda et al. 2001). And they concluded prolonged healing periods may be required for the cases using PHA. Reducing time of procedure has been considered beneficial for patients; however, further studies for verification of each material at each time point will be necessary to confirm the protocol. Since the preoperative bone height, systemic conditions and other related parameters are not constant in clinical cases, densities of calcified tissue and resisual hydroxyapatite will be different even with the same materials and protocols. Therefore, it can be complicating to compare the clinical outcomes directly between different materials. Currently, hydroxyapatite xenoglaft and alloplast are common bone substitutes for alveolar augmentation clinically (Kolerman et al. 2012; Boyne 1980; Chaves et al. 2012). Although there has been literatures describing results of using xenograft (Cassetta et al. 2014; De Souza Nunes 2011; Lambert et al. 2013; de Vicente et al. 2010; Carlo Mangano et al. 2006; Orsini et al. 2005; Stevens et al. 2005; Browaeys et al. 2007), only some reports have been published about clinical investigation for alloplast (Kolerman et al. 2012; Minamiguchi et al. 2008). In this study, sinus augmentation with hydroxyapatite alloplast was performed and evaluated.

Micro-CT is useful for evaluating bone parameters but does not identify new bone or bone remodeling (Huang et al. 2013; Kuhl et al. 2013). In contrast, histology can reveal cell morphology, new bone or even osteoid (Proussaefs et al. 2003; Ewers et al. 2004; Groeneveld et al. 1999); however, it does not permit images of the threedimensional architecture. In this study, analyses by both histology and micro-CT enabled clarified bone remodeling in the same sections from diverse perspectives. The results obtained from micro-CT revealed the BV/ TV reached only around $30 \%$ and hydroxyapatite residue was $1.2 \%$ in average, these alone could not optimize osseointegration of the implants but, from histology, could explain the active bone remodeling and bone formation in the grafted region such as mature osteoclasts, activated thickened osteoblasts with numerous secretory vesicles, and extensive vascularization indicated continuous active bone remodeling.

Although the drilling depth by trephine burs was around $6 \mathrm{~mm}$ underneath the $2 \mathrm{~mm}$ thick cortical bone, the height of harvested columnar bone samples was approximately $4-5 \mathrm{~mm}$. Therefore, bone formation at the interface of the sinus membrane and grafted bone was not evaluated in this study. However, this result might indicate the new bone generated from the residual bone such as alveolar ridge, septal wall and maxillary tuberosity due to location of the hydroxyapatite residues and the blanching morphology of the newly formed bone from this original bone. However, there is a repot of possibility

Table 1 Summary of the micro-CT analysis for native bone (B) + hydroxyapatite (HA) and HA residue

\begin{tabular}{|c|c|c|c|c|c|c|c|c|c|c|}
\hline & $\begin{array}{l}\text { "TV" } \\
{\left[\mathrm{mm}^{3}\right]}\end{array}$ & $\begin{array}{l}\text { "BV" } \\
{\left[\mathrm{mm}^{3}\right]}\end{array}$ & $\begin{array}{l}\text { “BS" } \\
{\left[\mathrm{mm}^{2}\right]}\end{array}$ & $\begin{array}{l}\text { “BS/BV" } \\
{[1 / \mathrm{mm}]}\end{array}$ & $\begin{array}{l}\text { “BV/TV" } \\
\text { [\%] }\end{array}$ & $\begin{array}{l}\text { "Tb.Th" } \\
\text { [urn] }\end{array}$ & $\begin{array}{l}\text { “Tb.N” } \\
\text { [1/mm] }\end{array}$ & $\begin{array}{l}\text { "Tb.Sp" } \\
\text { [urn] }\end{array}$ & $\begin{array}{l}\text { "Tb.Spac" } \\
\text { [urn] }\end{array}$ & “D” \\
\hline \multicolumn{11}{|l|}{$B+H A$} \\
\hline Subject 1 & 6.439 & 2.063 & 45.788 & 22.190 & 32.0 & 90.130 & 3.556 & 191.103 & 281.232 & 2.254 \\
\hline Subject 2 & 12.831 & 4.584 & 120.607 & 26.313 & 35.7 & 76.008 & 4.700 & 136.772 & 212.780 & 2.358 \\
\hline Subject 3 & 13.563 & 2.796 & 74.651 & 26.698 & 20.6 & 74.912 & 2.752 & 288.453 & 363.365 & 2.215 \\
\hline \multicolumn{11}{|l|}{$\mathrm{HA}$} \\
\hline Subject 1 & 6.439 & 0.132 & 9.396 & 71.169 & 2.1 & 28.102 & 0.730 & 1342.363 & 1370.465 & 2.033 \\
\hline Subject 2 & 12.831 & 0.197 & 18.987 & 96.247 & 1.5 & 20.780 & 0.740 & 1330.835 & 1351.615 & 1.888 \\
\hline Subject 3 & 13.563 & 0.015 & 1.645 & 107.744 & 0.1 & 18.562 & 0.061 & $16,474.093$ & $16,492.655$ & 1.474 \\
\hline
\end{tabular}

$T V$ tissue volume, $B V$ bone volume, $B S$ bone surface, $T b . T h$ trabecular thickness, $T b . N$ trabecular number, Tb.Sp trabecular separation, $T b . S p a c$ trabecular spacing, $D$ fractal dimension 
of bone formation along the sinus membrane(Sohn et al. 2011), thus, further investigations are required to confirm the mechanisms of bone formation in the grafted sinus.

\section{Conclusion}

Histological and micro-CT findings in bone cores at estimated implant placing sites indicate bone regeneration within and between porous hydroxyapatite allopllast 6 month after maxillary sinus augmentation.

\section{Additional files}

Additional file 1. Horizontal rotation movie corresponding to Fig. 3a. Additional file 2. Horizontal rotation movie corresponding to Fig. 3b. Additional file 3. Horizontal rotation movie corresponding to Fig. 3c.

\section{Abbreviations}

$\mathrm{CT}$ : computed tomography; TV: tissue volume; BV: bone volume; BS: bone surface; Tb.Th: trabecular thickness; Tb.N: trabecular number; Tb.Sp: trabecular separation; Tb.Spac: trabecular spacing; D: fractal dimension; $\mathrm{V}$ : vein; $\mathrm{A}$ : artery; NB: new bone; OB: osteoblast; OC: osteoclast; HA: hydroxyapatite; FB: fibroblast; BC: blood cell.

\section{Authors' contributions}

HN treated the patients, acquisition of the data, and drafting the manuscript. SK conception of the study and revising the manuscript. NT design of the study. EO technical support and data analysis. MA acquisition of the data and data analysis. SK approve the manuscript. HK final approval of the manuscript. All authors read and approved the final manuscript.

\section{Author details}

${ }^{1}$ Department of Prosthodontics and Oral Implantology, Dental Hospital, Iwate Medical University, 19-1 Uchimaru, Morioka, Iwate, Japan. ${ }^{2}$ Department Oral Implantology and Regenerative Dental Medicine, Graduate School of Medical and Dental Sciences, Tokyo Medical and Dental University, 1-5-45, Bunkyo-ku, Yushima, Tokyo 113-8510, Japan.

\section{Acknowledgements}

The authors thank Dr. Michiko Suzuki, Oral Implantology and Regenerative Dental Medicine, Tokyo Medical and Dental University, for technical advice, and Dr. Hidemichi Kihara and Dr. Kyoko Takafuji, Prosthodontics and Oral Implantology, Iwate Medical University, for clinical support.

\section{Financial support and competing interests}

This research was supported by a Grant (\#24659871) from the Japanese Society for the Promotion of Science to Iwate Medical University. The authors declare that they have no competing interests.

Received: 13 July 2015 Accepted: 16 February 2016

Published online: 02 March 2016

\section{References}

Boyne PJJR (1980) Grafting of the maxillary sinus floor with autogenous marrow and bone. J Oral Surg 38:613-616

Browaeys H, Bouvry P, De Bruyn H (2007) A literature review on biomaterials in sinus augmentation procedures. Clin Implant Dent Relat Res 9:166-177

Campana V, Milano G, Pagano E, Barba M, Cicione C, Salonna G et al. (2014) Bone substitutes in orthopaedic surgery: from basic science to clinical practice. J Mater Sci Mater Med
Carlo Mangano M, Antonio Scarano D, Giovanna lezzi D, Giovanna Orsini D, Vittoria Perrotti D, Francesco Mangano D et al (2006) Maxillary sinus augmentation using an engineered porous hydroxyapatite: a clinical,histological, and Transmission electronmicroscopy study in man. J Oral Implantol XXXII: 122-131

Cassetta M, Perrotti V, Calasso S, Piattelli A, Sinjari B, lezzi G (2014) Bone formation in sinus augmentation procedures using autologous bone, porcine bone, and a 50: 50 mixture: a human clinical and histological evaluation at 2 months. Clin Oral Impl Res (in press)

Chaves MD, de Souza Nunes LS, de Oliveira RV, Holgado LA, Filho HN, Matsumoto MA et al (2012) Bovine hydroxyapatite (Bio-Oss((R))) induces osteocalcin, RANK-L and osteoprotegerin expression in sinus lift of rabbits. J Craniomaxillofac Surg 40:e315-320

De Souza Nunes LS, De Oliveira RV, Holgado LA, Nary Filho H, Ribeiro DA, Matsumoto MA (2011) Use of bovine hydroxyapatite with or without biomembrane in sinus lift in rabbits: histopathologic analysis and immune expression of core binding factor 1 and vascular endothelium growth factor. J Oral Maxillofac Surg 69:1064-1069

de Vicente JC, Hernandez-Vallejo G, Brana-Abascal P, Pena I (2010) Maxillary sinus augmentation with autologous bone harvested from the lateral maxillary wall combined with bovine-derived hydroxyapatite: clinical and histologic observations. Clin Oral Impl Res 21:430-438

Ewers R, Goriwoda W, Schopper C, Moser D, Spassova E (2004) Histologic findings at augmented bone areas supplied with two different bone substitute materials combined with sinus floor lifting: report of one case. Clin Oral Impl Res 15:96-100

Fassina L, Saino E, Sbarra MS, Visai L, De Angelis MG, Magenes G et al (2010) In vitro electromagnetically stimulated SAOS-2 osteoblasts inside porous hydroxyapatite. J Biomed Mater Res, Part A 93:1272-1279

Groeneveld vdBJ EH, Holzmann P, ten Bruggenkate CM, Tuinzing DB, Burger EH (1999) Mineralization processes in demineralized bone matrix grafts in human maxillary sinus floor elevations. J Biomed Mater Res 48:393-402

Huang HL, Hsu JT, Chen MY, Liu C, Chang CH, Li YF et al (2013) Microcomputed tomography analysis of particular autogenous bone graft in sinus augmentation at 5 months: differences on bone mineral density and 3D trabecular structure. Clin Oral Invest 17:535-542

Karabuda C, Ozdemir O, Tosun T, Anil A, Olgaç V (2001) Histological and clinical evaluation of 3 different grafting materials for sinus lifting procedure based on 8 cases. J Periodontol 72:1436-1442

Kolerman R, Goshen G, Joseph N, Kozlovsky A, Shetty S, Tal H (2012) Histomorphometric analysis of maxillary sinus augmentation using an alloplast bone substitute. J Oral Maxillofac Surg 70:1835-1843

Kuhl S, Brochhausen C, Gotz H, Filippi A, Payer M, d'Hoedt B et al (2013) The influence of bone substitute materials on the bone volume after maxillary sinus augmentation: a microcomputerized tomography study. Clin Oral Invest 17:543-551

Lambert F, Lecloux G, Leonard A, Sourice S, Layrolle P, Rompen E (2013) Bone regeneration using porous titanium particles versus bovine hydroxyapatite: a sinus lift study in rabbits. Clin Implant Dent Relat Res 15:412-426

Liu D-M (1996) Fabrication and characterization of porous hydroxyapatite granules. Biomaterials 17:1955-1957

Minamiguchi S, Takechi M, Yuasa T, Momota Y, Tatehara S, Takano H, Miyamoto Y, Satomura K, Nagayama M (2008) Basic research on aw-AC/PLGA composite scaffolds for bone tissue engineering. J Mater Sci Mater Med 19:1165-1172

Moller B, Acil Y, Birkenfeld F, Behrens E, Terheyden H, Wiltfang J (2014) Highly porous hydroxyapatite with and without local harvested bone in sinus floor augmentation: a histometric study in pigs. Clin Oral Impl Res 25:871-878

Orsini GTT, Scarano A, Degidi M, Perrotti V, Piccirilli M, Piattelli A (2005) Maxillary sinus augmentation with Bio-Oss particles: a light, scanning, and transmission electron microscopy study in man. J Biomed Mater Res B Appl Biomater 1:448-457

Periklis Proussaefs JL, Jay Kim (2003) Effects of sealing the perforated sinus membrane with a resorbable collagen membrane: a pilot study in humans. J Oral Implantol 235-241 
Phipps MC, Clem WC, Grunda JM, Clines GA, Bellis SL (2012) Increasing the pore sizes of bone-mimetic electrospun scaffolds comprised of polycaprolactone, collagen I and hydroxyapatite to enhance cell infiltration. Biomaterials 33:524-534

Sohn DS, Moon JW, Lee WH, Kim SS, Kim CW, Kim KT, Moon YS (2011) Comparison of new bone formation in the maxillary sinus with and without bone grafts: immunochemical rabbit study. Int J Oral Maxillofac Implants 26:1033-1042

Stevens MM, Marini RP, Schaefer D, Aronson J, Langer R, Shastri VP (2005) In vivo engineering of organs: the bone bioreactor. Proc Natl Acad Sci USA 102:11450-11455
Tatum H Jr (1986) Maxillary and sinus implant reconstructions. Dent Clin North Am 30:207-229

Yoshikawa H, Tamai N, Murase T, Myoui A (2009) Interconnected porous hydroxyapatite ceramics for bone tissue engineering. J R Soc Interface 6(Suppl 3):S341-348

Yuasa T, Miyamoto Y, Ishikawa K, Takechi M, Momota Y, Tatehara S, Nagayama $M$ (2004) Effects of apatite cements on proliferation and differentiation of human osteoblasts in vitro. Biomaterials 25:1159-1166

\section{Submit your manuscript to a SpringerOpen ${ }^{\odot}$ journal and benefit from:}

- Convenient online submission

- Rigorous peer review

- Immediate publication on acceptance

- Open access: articles freely available online

- High visibility within the field

- Retaining the copyright to your article

Submit your next manuscript at $>$ springeropen.com 